\title{
A Foreign Language Learning Application using Mobile Augmented Reality
}

\author{
Florentin - Alexandru DIȚĂ \\ Bucharest University of Economic Studies, Bucharest, Romania \\ alexandru.dita@csie.ase.ro
}

In this paper is described a foreign language learning application using mobile augmented reality based on gamification method and text recognition. The mobile augmented reality is a technology that extends the real world elements with $2 D$ or $3 D$ computer generated objects and lets the users interact with them. A Gamification system is based on different mechanisms that increase the motivation of students, due to the impact that videogames have in their emotional, cognitive and social areas. The proposed solution applies Optical Character Recognition technique, using the camera of the mobile device, in order to identify the text written on a card. The implementation combines the features of gamification system and mobile augmented reality in order to make the learning process more easy and fun. This paper aims to present the results after testing the foreign language learning application in different scenarios.

Keywords: Gamification, M-Learning, Mobile Augmented Reality, Optical Character Recognition, Virtual Object

1 Introduction

Nowadays, the education institutes already use in the process of learning the advantages of mobile learning technology, such as portability (the students have easy access at any time to information), the use of digital assets (audio and video files) and huge amount of textual information. This study proposes a new system approach for improving the learning process by using Mobile Augmented Reality. The features of this technology stimulate the learning environment and consider that the student's motivation is the most important factor in learning.

The existing literature [1] highlights two different approaches for augmented reality (AR). Firstly, the authors consider that AR is a system which has the following characteristics: Combines real and virtual; Interactive in real time; Registered in 3-D. Secondly [1] considers Augmented Reality part of Virtual Continuum. This context is composed of two elements: Real Environment - which contains all representations from real life and Virtual Environment - which combines different elements from virtual life. ARs are considered closer to reality than virtual environment because this type of systems are supposed to extend the real objects with virtual elements.
According to [2] Augmented Reality is a technology that extends the real world elements with $2 \mathrm{D}$ or $3 \mathrm{D}$ computer generated objects and lets the users interact with them. Also, the authors present three types of devices which can be used to view the result of AR technology: a Head Mounted Display (HMD), a mobile device - with at least one back camera and a desktop or a laptop computer.

Similarly, [2] considers mobile learning technology as an alternative teaching resource because the users have easy access in real time to information. Also, using mobile devices in the learning process two main problems of traditional learning are solved: the location and time. Likewise, [1] considers that mobile learning is a type of eLearning without restrictions of time and space and this technology improves the quality of classical learning environments, but does not replace them.

Other researches, such as [3] and [4] highlight the benefits of using a Mobile Augmented Reality system based on tracking objects. In [3] is proposed a mobile augmented reality system based on visual recognition. According to the authors the visual recognition consists into tracking objects identified in real world with the camera of the mobile device and augmenting the scene with virtual objects created by MAR technology. Similarly, [4] presents a 
Mobile Augmented Reality system bases on $3 \mathrm{D}$ registration technology. According to the authors the $3 \mathrm{D}$ registration represents the tracking of the position and pose of the mobile device in the real time and real life and finally use these information to insert in the real world the virtual scene created by MAR. The $3 \mathrm{D}$ registration technology is based on two type of approaches: markers and natural features. Recently, the 3D registration for MAR uses GPS and sensors from mobile devices to provide different information about position and pose of the camera and combine them with computer vision to improve the speed and accuracy of the system.

Other studies, such as [5] propose a makerbased Mobile Augmented Reality solution. The application is used by a Vocational Educational and Training (VET) program for supporting the maintenance process of a car. The authors define VET as "education programs that are designed for learners to acquire the knowledge, skills and competencies specific to a particular occupation, trade or class of occupations or trades"(p. 49-50). Moreover, in [5] is presented an AR application used by VET students for basic mathematics skills, which is based on videogames.

More studies, such as [6] present a Mobile Augmented Reality solution for learning French language which is based on gamification didactic method. The author, Bernadette Perry, describes gamification as "using gamebased mechanics, aesthetics and game thinking to engage people, motivate action, and promote learning" (p. 2309). [6] suggests that the features of MAR technology, such as 3D registration or visual recognition, improve the benefits of gamification method.

Moreover, the authors of [7] consider that "gamification is the use of the game design elements in non-game contexts" in order to improve the children's study motivation and give them the opportunity for experimenting in safe environments.

In this paper is proposed a mobile augmented reality system for learning a foreign language, based on text recognition and gamification didactic method. The first step of the implemented solution is to identify the text written on a card, applying Optical Character Recognition technique, using the camera of the mobile device. Afterwards, the identified word will be displayed on the device's screen, augmented with virtual 2D objects. Finally, the application allows the pronunciation of the object spelling in a language which is selected by the user. The target users of the prototype presented in this paper are the children between 5 and 10 years old that want to learn new words from one of the available foreign languages. From an economical point of view, the proposed solution offers the benefit of reducing the additional costs, which were caused by the buying of different books or learning materials, in order to start the learning process.

The paper is organized as follows. Section 2 analyzes the architecture of the proposed application. The implementation of the prototype is shown in Section 3. Section 4 presents the results obtained by using the solution and describes the future work.

\section{Application Architecture}

The purpose of this paper is to present a foreign language learning application using mobile augmented reality based on gamification didactic method and text recognition. According to [6], 35 is the average age of the gamers and $97 \%$ of adolescents play video games. Moreover, as stated in [8] a gamification system increases the motivation of pupils, due to the impact that videogames have in their emotional, cognitive and social areas. Consequently, learning a foreign language becomes more easy and fun using a gamification approach.

The text recognition system is needed, considering that the inputs of the application consist in cards containing words from different categories. This approach is based on applying a similarly Optical Character Recognition (OCR) technique, such as [9] to detect the written text, and an algorithm to match the results of OCR with available words.

The proposed architecture for foreign language learning application using mobile augmented reality, is shown in Figure 1. 


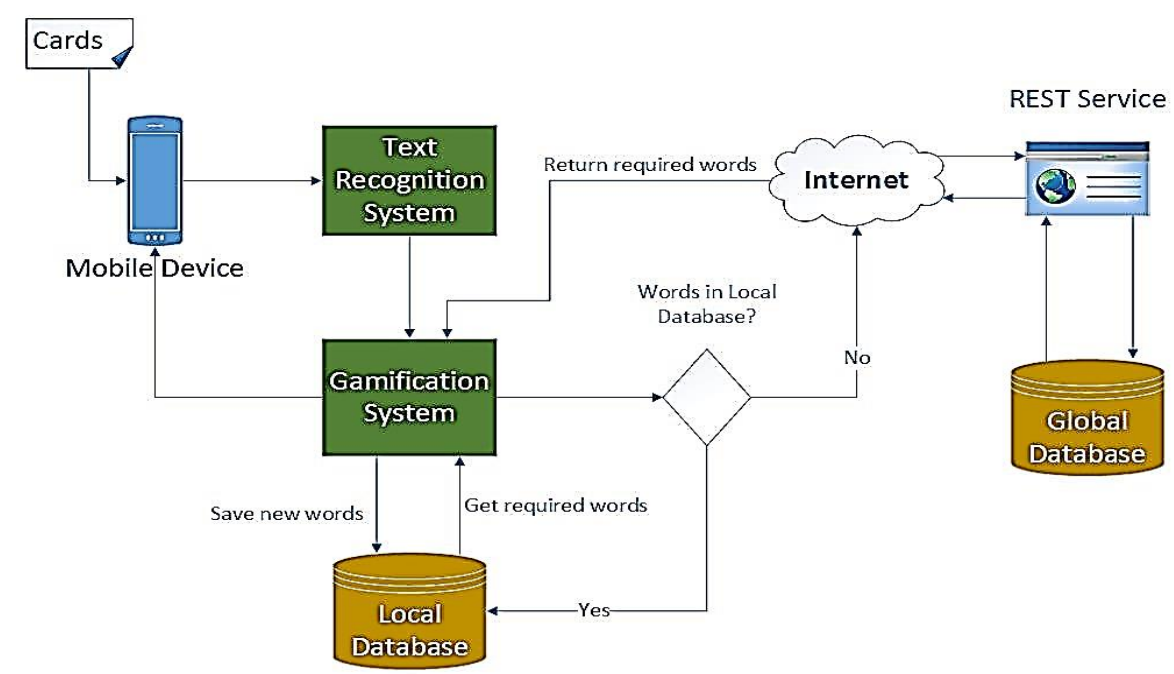

Fig. 1. Architecture of foreign language learning application using mobile augmented reality

The input of the application consists in already prepared cards containing words from different categories, such as: animals, fruits, vegetables. The current solution supports translations from Romanian in one of the following languages: English, French and Italian. The mobile solution accesses the device camera to get frames with the cards. For every identified frame the text recognition process is applied in order to detect the written word. After the detection process is successfully finished, the results are sent to the gamification process. Firstly, in this stage, the application checks if the corresponding virtual objects of the previous identified words are already saved in the local database, before augmenting them on the device's screen. In case they are not found, the application calls the REST Service in order to get the virtual objects from the global database. According to [3] a REST solution is a service or an application that can be used by any device which executes HTTP calls. The virtual objects, which are returned after querying the REST Service, are then saved by the application in the local database. Secondly, the gamification process uses the corresponding virtual objects that have been stored in the local database in order to draw them on the device's display.

The REST Service contains methods that allow to retrieve the following needed information: the available categories and the stored data on the existing words. This data represents the virtual objects that are used in the gamification system and consist of: the corresponding 2D images of the available words and the translations of the Romanian texts in English, French and Italian. The global database contains five tables in order to manage the functionalities of the presented mobile augmented reality application. On the other hand, the local database defines two tables that store the virtual objects of the selected category. The records from this table are replaced every time when the selected category is changed in order to reduce the needed space on the mobile device.

\section{Implementation}

The mobile augmented reality application is developed with Java programming language using Android SDK and tess-two library. Tess-two is an open source library based on Tesseract OCR Engine and Leptonica Engine processing image which permits words identification from different languages. The current application is composed of two systems: Text Recognition and Gamification.

\subsection{Text Recognition System}

Text Recognition system is based on an engine class which uses the tess-two library in order to get the written text and an algorithm for matching them with available words from the selected category. The matching algorithm 
consists in checking if a least $80 \%$ of the characters from a text returned by the OCR engine are identically and in the same order with characters of an available word from the selected category.

In the OCR engine method, which communicates with the tess-two library, two types of variables are defined in order to speed up the processing done with the OCR technique. The first type, called VAR_CHAR_WHITELIST, contains the available characters of the detection process from the used library. The second type of variable is the VAR_CHAR_BLACKLIST which defines the unavailable characters of the detection process. Also, the application checks if the difference between the length of the text detected by the recognition engine and the length of an available word from a selected category is $-1,0$ or 1 before calling the checkWord () method. In Figure 2, a workflow of the Text Recognition System is presented.

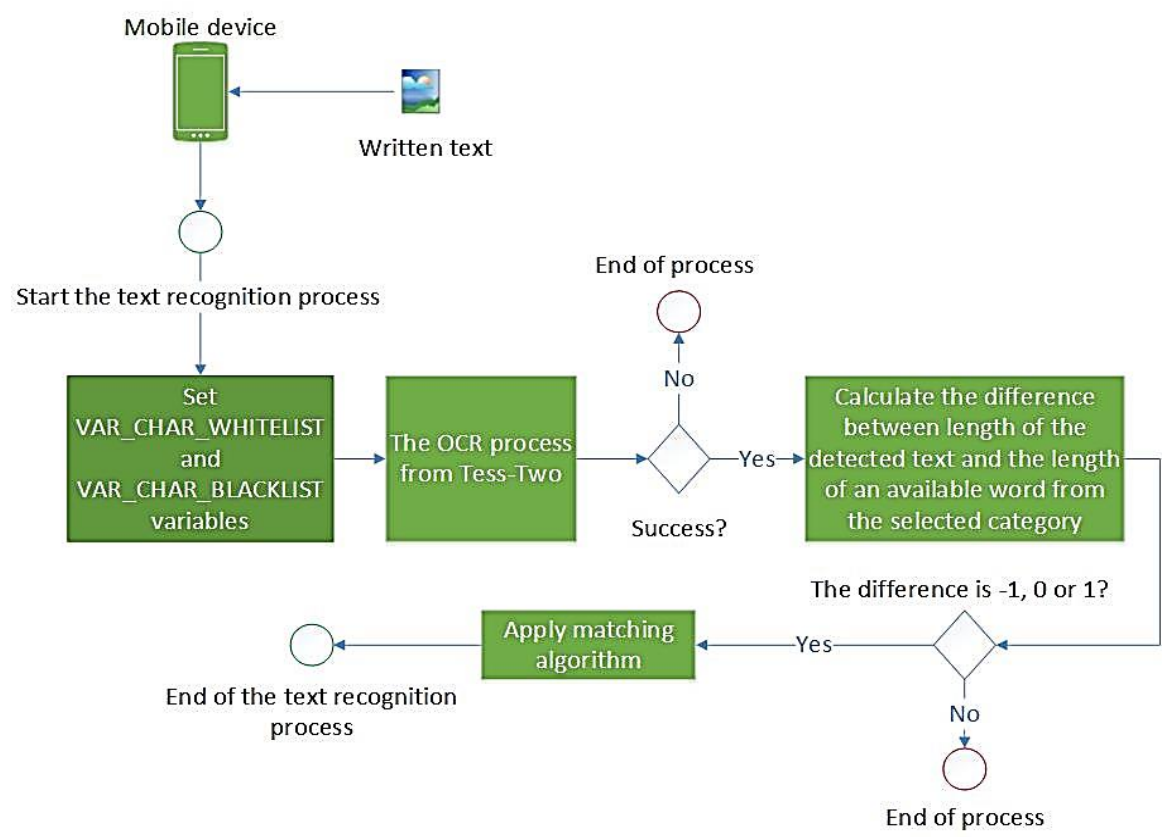

Fig. 2. Workflow of the Text Recognition System

The next listing shows a part of the check- algorithm.

Word() method that implements the matching

Listing 1. Matching algorithm method

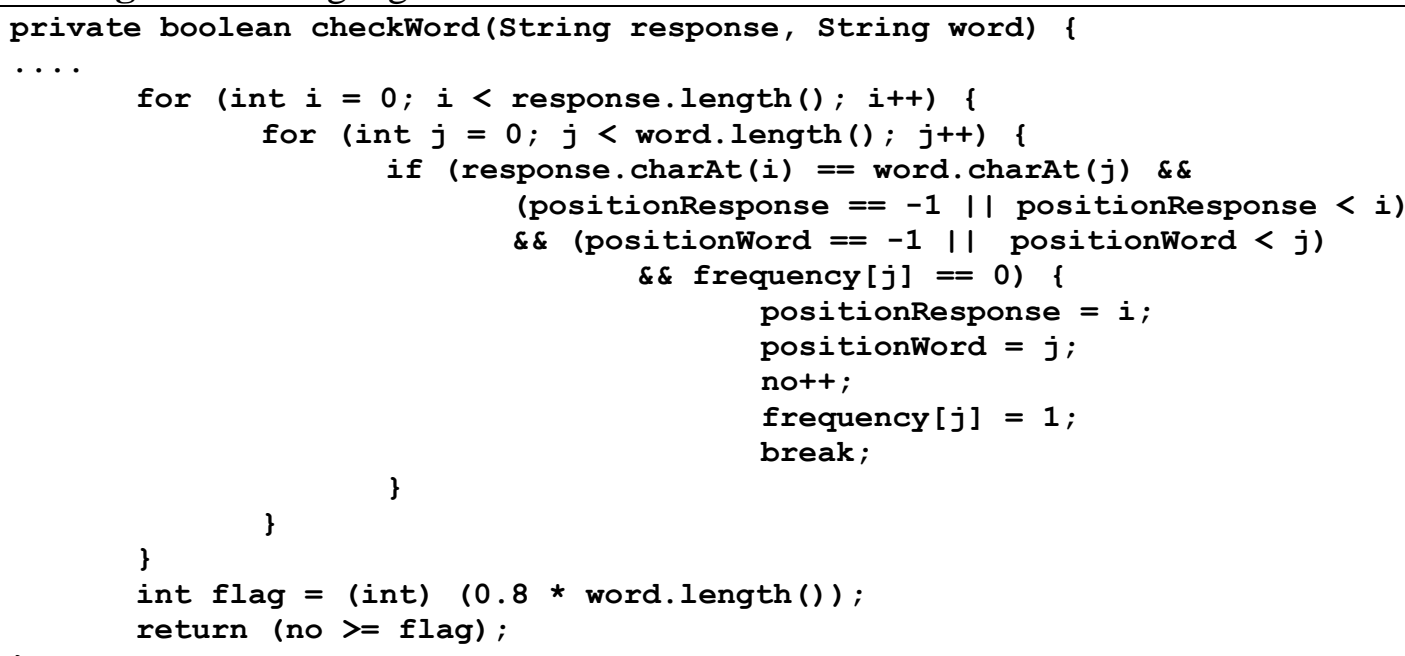


The checkWord() method uses the variables positionResponse, positionWord and frequency in order to check if the characters of the detected text are in the same order as the characters of an available word from the system.

\subsection{The Gamification System}

Gamification System is based on a controller class which uploads the virtual objects corresponding to the written words detected by the Text Recognition System from the database, an engine class which draws them on the mobile screen and a translation system which uses Text-To-Speech (TTS) and the language chosen by the user.

In the first section of the Gamification System the user has to select one from the following three types of foreign languages in which the detected word is translated: English, French or Italian.

The uploading process of the virtual objects consists in two steps: firstly, the system search them in the local database, which is installed on the mobile device. Secondly, if the result of previous querying is empty then the system gets the virtual objects from the global database using a REST Service.

In the next step, if the uploading process is successfully finished, the Gamification System uses methods of the drawing engine in order to augment the virtual objects on the mobile device. This part includes the creation of the 2D image, the calculation of its coordinates in the drawing area and the visual components which are used for starting the translation (microphone image) and changing the objects between them (left right arrows).

Finally, the translation system consists in methods that are responsible for taking over the word from the displayed virtual object, based on the language that is chosen by the user before starting the uploading process, and a method that uses the Google Text-to-Speech API functionality in order to pronounce the translated word.

In Figure 3, is presented a workflow of the Gamification System.

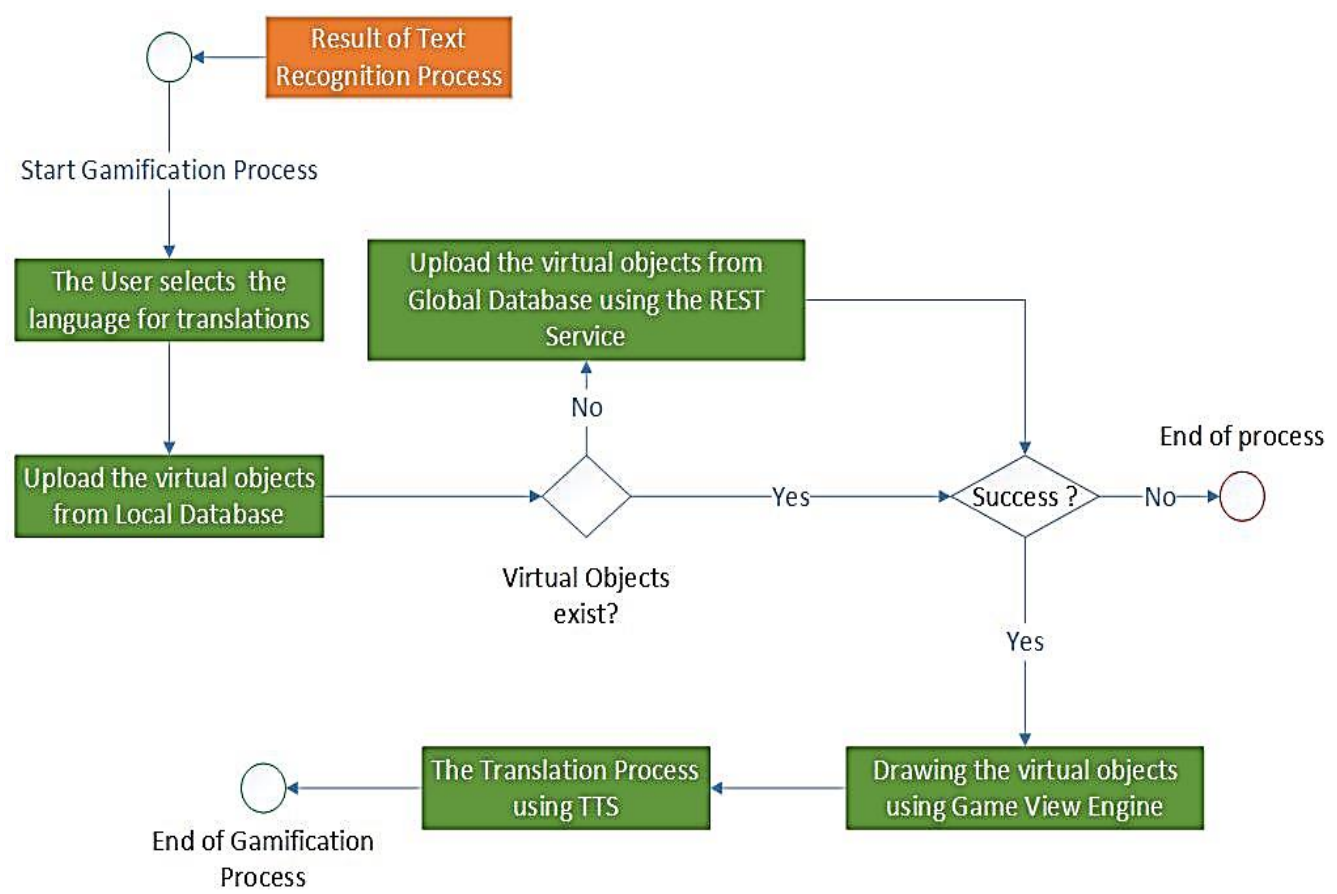

Fig. 3. Workflow of the Gamification System

The implementation of the Gamification System is composed of the following three components:

- the interaction with the local database;
- the drawing of the stored virtual objects;

- the communication with the REST Service;

- the translation process with TTS. 
The first part consists in using a SQLite Database in order to store and retrieve the virtual objects from the REST Service API. The component implements a controller class, which has the necessary methods for creating the SQLite Database. This is derived from the abstract class SQLiteOpenHelper. According to [10], the SQLiteOpenHelper contains methods that allow to create the database and obtains an instance of the SQLiteDatabase class. The previous class includes specialized methods for adding, deleting and updating records. Also, the SQLiteDatabase exposes methods that permit direct execution of SQL commands such as: execSQL(), used for the insert commands and rawQuery(), used for select commands. Moreover, the component contains a second class that defines the methods for data manipulation using the presented controller class and an instance of the SQLiteDatabase class.
The component that does the drawing of the stored virtual objects is based on a game view engine. This extends SurfaceView class which allows to augment on the mobile device's screen different elements, such as: pictures, texts, buttons, lines, rectangles etc. The virtual objects are encapsulated in a class that has the following fields: a Bitmap that contains a sprite image, the width and the height of the image, the number of rows, the number of columns and the position where the virtual object must be drawn. A sprite is a static image or an animated graphic that is used to create $2 \mathrm{D}$ videogames. The game view engine overrides the onDraw() method from SurfaceView in order to draw on the mobile screen the virtual objects based on the previous class. The Listing 2 shows the implementation of onDraw() method.

Listing 2. Drawing of a virtual object

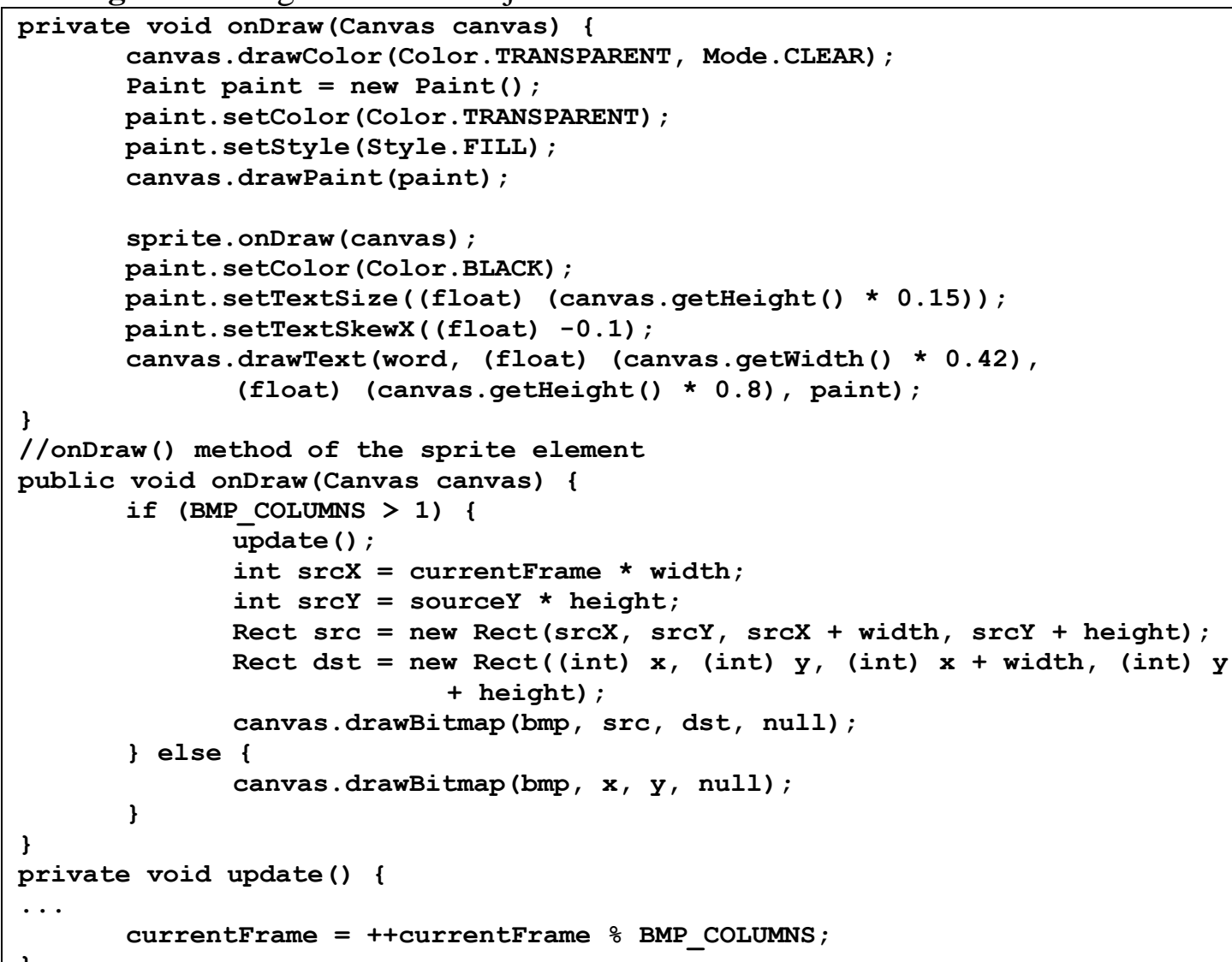

The third component of the system is repreHTTP calls over the REST Service in order to sented by an AsyncTask class which executes 
get the required virtual objects. The AsyncTask class implements the doInBackground() method that interacts with the REST Service functionalities. Firstly, this method executes a HTTP request to the service in order to get the virtual objects from a specified category. The response of the REST Service call is a JSON object that contains the needed information. Secondly, the doInBackground() method uses the GSON library in order to transform the JSON object into a corresponding Java class. Listing 3 presents a part of the REST Service engine.

Listing 3. The communication with the REST Service

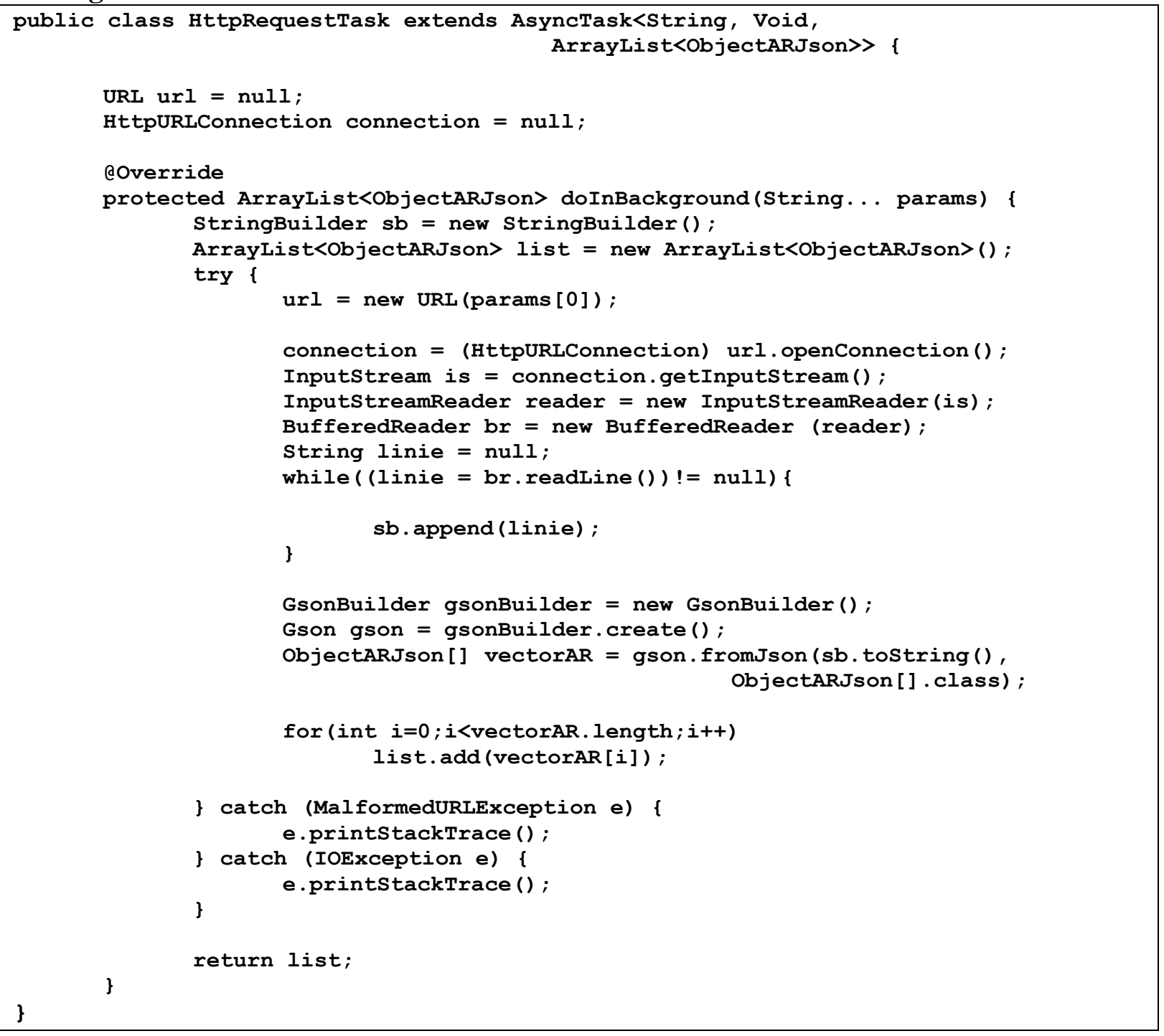

The REST Service is developed with Java programming language using SpringBoot framework in order to create a Web application based on a REST architecture. The REST Service is composed of methods that allow communication with an Oracle Database, that stores information about the virtual objects. The last element of the Gamification System is a translation process based on Text-ToSpeech (TTS) functionality. According to
[11], the core functionality of TTS is "text processing that functions as the producer of syllabic speech units to be used in the generation of human-like speech". This process contains two methods for translating the identified words by Text Recognition System, namely: setCurrentTranslations() and speak(). 
The first method creates a HashMap object which contains the translations of the displayed virtual object in each of the three possible language (English, French, Italian). Translated words, which are used in setCurrentTranslations(), are retrieved from the da- tabase together with the data of the virtual objects. Also, this information contains the ISO codes of the corresponding languages which are used as keys of the map elements. Listing 4 shows a part of the setCurrentTranslations() method.

Listing 4. Current translations method

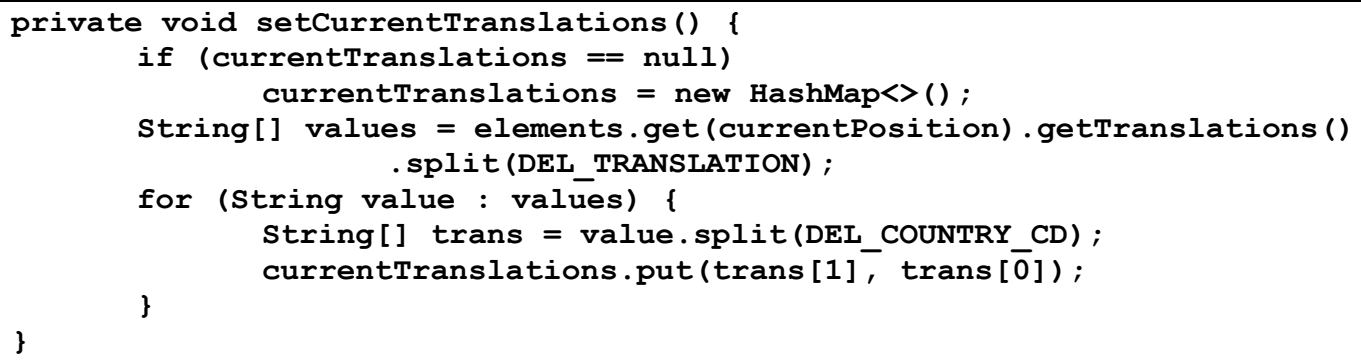

The speak() method is used for calling the speaking functionality of the TTS object, based on the language selected by the user in the beginning of the Gamification System and the result of the setCurrentTranslations() method. This method gets the right translated word from the HashMap object using the selected language as a key and gives the corresponding element to the TTS in order to pronounce it.

\section{Results and Discussions}

The result of testing the proposed foreign language learning application using mobile augmented reality is presented in Table 1 and Figure 4-7. Table 1 contains the information obtained by using the Text Recognition System in the following scenario: given four type of cards, having different size of the written text and containing words from the application known categories or outside of them, placed at various distances from the mobile device.

Table 1. The Result of Text Recognition System Tests

\begin{tabular}{|l|l|c|c|c|c|}
\hline Type of card & Font size & $\begin{array}{l}\text { Number of } \\
\text { known } \\
\text { words }\end{array}$ & $\begin{array}{c}\text { Distance } \\
\text { (cm) }\end{array}$ & $\begin{array}{c}\text { Average of } \\
\text { frames processed }\end{array}$ & $\begin{array}{c}\text { Average of } \\
\text { duration } \\
\text { (seconds) }\end{array}$ \\
\hline $\begin{array}{l}\text { Card with 7 } \\
\text { words }\end{array}$ & $\begin{array}{l}\text { Times } \\
\text { New Ro- } \\
\text { man 60 }\end{array}$ & 3 & 70 & 4 & 6.3 \\
\hline $\begin{array}{l}\text { Card with } \\
\text { one word }\end{array}$ & $\begin{array}{l}\text { Times } \\
\text { New Ro- } \\
\text { man 60 }\end{array}$ & 1 & 70 & 2.5 & 3.4 \\
\hline $\begin{array}{l}\text { Card with } \\
\text { one word }\end{array}$ & $\begin{array}{l}\text { Times } \\
\text { New Ro- } \\
\text { man 150 }\end{array}$ & 1 & 70 & 2 & 2.9 \\
\hline $\begin{array}{l}\text { Card with 7 } \\
\text { words }\end{array}$ & $\begin{array}{l}\text { Times } \\
\text { New Ro- } \\
\text { man 60 }\end{array}$ & 3 & 140 & 7.8 & 10.5 \\
\hline
\end{tabular}

Also, for each type of card, the system was executed 10 times in order to calculate the averages of processed frames and duration needed to detect all the correct words. As shown in
Table 1, we can see that the application needs 6.3 seconds to identify three words, after processing four frames from the device's camera. 
On the other hand, for only one word the system needs 3.4 seconds at an average of 2.5 processed frames. Consequently, the duration of the detection system is influenced by the number of words. Also, comparing the first and the last record from Table 1, we can see that the performance of the text recognition system is impacted directly by the distance between the mobile device and the card, given that the others variables remain the same. Moreover, analyzing the second and the third row from Table 1, we can conclude that the system performance is improved by the font size of the words.

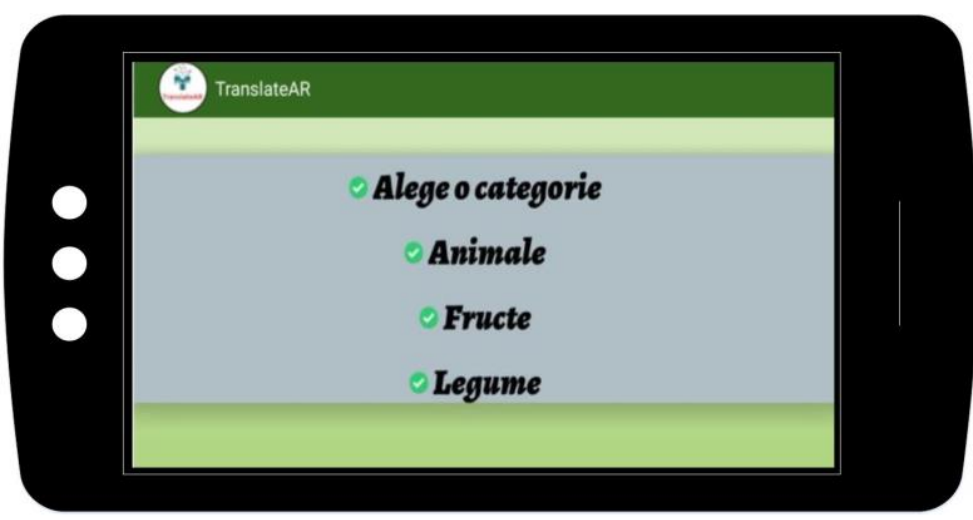

Fig. 4. The available words category

In Figure 4 is displayed a drop down list, the user having the possibility to choose from its items, one category of words that the application will use to create the augmented scenes on the device's screen.

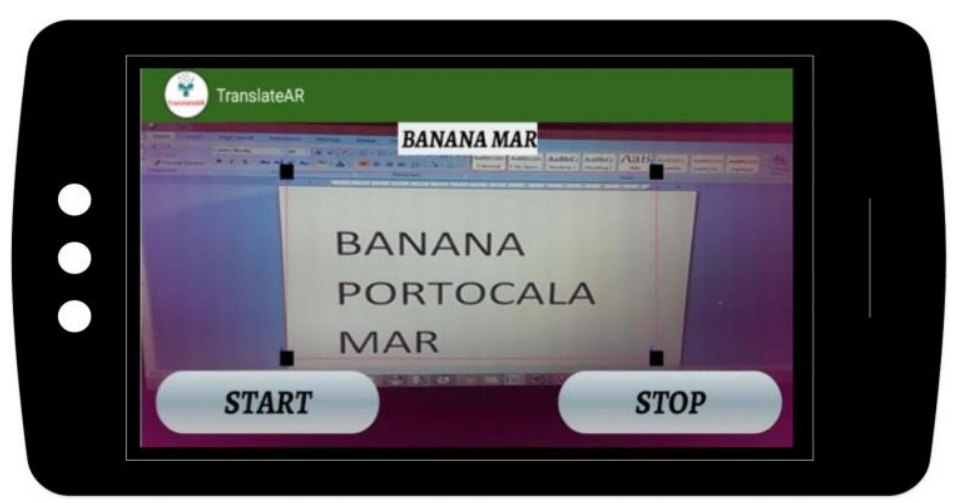

Fig. 5. The detection part of Text Recognition System

Figure 5 presents what the camera is capturing just before the matching algorithm to display the results. This part represents the result of calling the OCR engine method, which communicates with the tess-two library in order to identify the written text. The "Stop" button fires the event that sends the previous result to the last element of the text recognition system, namely, the matching algorithm. 


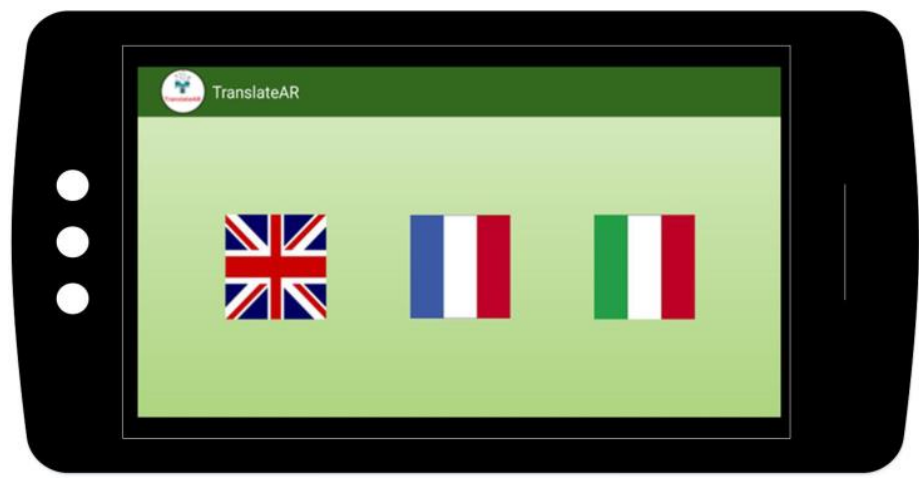

Fig. 6. The foreign language selection

After the detection of the written words has finished, the application shows the menu from Figure 6, providing the user with the chance to choose the translation language, which represents the first step of the gamification system. Also, in this section is called the uploading process of the corresponding virtual ob-

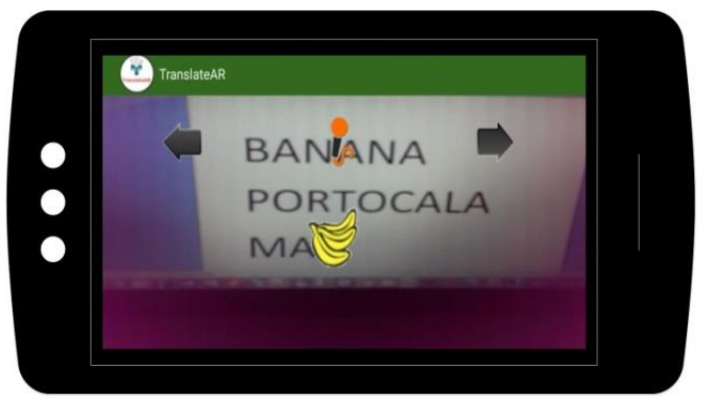

Fig. 7. The result of gamification system

Finally, in Figure 7 is showed the result of the using the onDraw() method from the game view engine which is responsible with drawing of the corresponding virtual objects on the device screen. Also, this section contains the translation process using the Text-to-Speech (TTS) which is the last element of the gamification system.

The microphone from the Figure 7 uses the speak() method of a TTS object in order to pronounce the translated word.

\section{Conclusions and Future Work}

In this paper was proposed a foreign language learning application using mobile augmented reality based on text recognition and gamification method. The text recognition system allows identifying the text written on a card by applying an Optical Character Recognition (OCR) technique and an algorithm to match jects of the identified words which is represented by the REST Service and the interaction with the locale database. Finally, after one flag from the Figure 6 is touched the system calls the setCurrentTranslations() method which is the first element of the translation process.

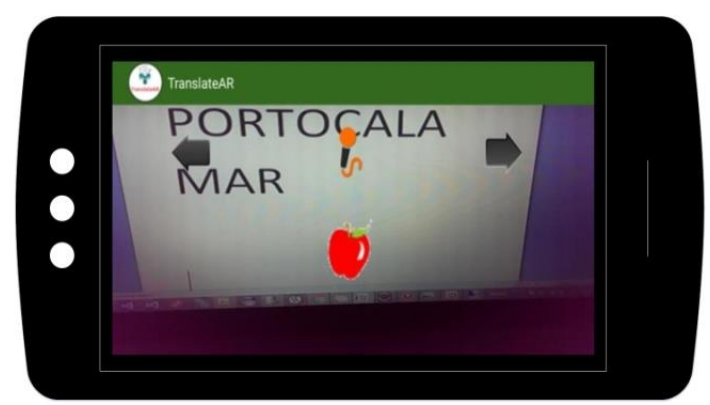

the results of OCR with available words. The analysis presented in previous section, suggests that the system is influenced either by the font size of the written text or the number of words from the application known categories or the distance between the card and the mobile device.

The gamification system designs and augments on the device screen the corresponding virtual objects of the words detected by the previous engine. Also, this system uses the Text-To-Speech functionality in order to pronounce the translation of the identified word by the text recognition system. Moreover, using a gamification system the motivation of pupils is increased, due to the impact that videogames have in their emotional, cognitive and social areas. Consequently, the presented 
mobile augmented reality application becomes more easy and fun implementing a gamification approach.

In future work, we want to improve the matching algorithm from the text recognition system in order to reduce the time necessary to detect the written words. Moreover, the extending of the current number of available categories and foreign language from the application is another objective of the future work.

Finally, mobile augmented reality system can be used to improve the users' knowledge regarding a foreign language in an interactive way because of that the application implements a gamification approach which makes the learning process easier.

\section{Acknowledgment}

Parts of this research have been published in the Proceedings of the $15^{\text {th }}$ International Conference on Informatics in Economy, IE 2016 [12].

\section{References}

[1] T. Roffmann and T. Friese, "Mobile Augmented Reality for Learning", Seminar: Mobile Learning winter semester 2011/2012 Computer-Supported Learning Research Group, 2012.

[2] S. Jamali, M. Shiratuddin, K. Wong and C. Oskam, "Utilising Mobile-Augmented Reality for Learning Human Anatomy", Procedia - Social and Behavioral Sciences - 7th World Conference on Educational Sciences, vol. 197, Athens, Greece 2015, pp. 659-668.

[3] J. Sanchez, E. Tello-Leal, J. CarreonGutierrez, V. Saldivar-Alonso and T. Guerrero-Melendez, "An Augmented Reality System Approach for Mobile Devices", International Journal of Latest Research in Science and Technology, vol. 2, no. 5, pp. 9-11, September-October 2013.

[4] P. Chen, Z. Peng, D. Li and L. Yang, "An improved augmented reality system based on AndAR", Journal of Visual Communication and Image Representation, vol. 37, pp. 63-69, May 2016.

[5] J. Bacca, S. Baldiris, R. Fabregat, Kinshuk and S. Graf, "Mobile Augmented Reality in Vocational Education and Training", Procedia Computer Science - 2015 International Conference Virtual and Augmented Reality in Education, vol. 75, Monterrey, Mexico, 2015, pp. 49-58.

[6] B. Perry, "Gamifying French Language Learning: A Case Study Examining a Quest-based, Augmented Reality Mobile Learning-tool", Procedia - Social and Behavioral Sciences - International Conference on New Horizons in Education, vol. 174, Paris, France, 2015, pp. 2308-2315.

[7] B. Müller, C. Reise and G. Seliger, "Gamification in Factory Management Education - A Case Study with Lego Mindstorms", Procedia CIRP - 12th Global Conference on Sustainable Manufacturing - Emerging Potentials, vol. 26, Johor Bahru, Malaysia, 2015, pp. 121-126.

[8] A. Domínguez, J. Saenz-de-Navarrete, L. de-Marcos, L. Fernández-Sanz, C. Pagés and J. Martínez-Herráiz, "Gamifying learning experiences: Practical implications and outcomes", Computers \& Education, vol. 63, pp. 380-392, April 2013.

[9] R. Smith, D. Antonova and D. Lee, "Adapting the Tesseract open source OCR engine for multilingual OCR", Proceedings of the International Workshop on Multilingual OCR - MOCR '09, Barcelona, Spain, 2009.

[10] P. Pocatilu, "Building Database-Powered Mobile Applications", Informatica Economica, vol. 16, no. 1, pp. 132-142, October 2012 .

[11] Ramli, Izzad et al. "An Improved Syllabification For A Better Malay Language Text-To-Speech Synthesis (TTS)". Procedia Computer Science - 2015 IEEE International Symposium on Robotics and Intelligent Sensors, vol. 76, Langkawi, Malaysia, 2015, pp. 417-424.

[12] F. A. Diță, "The prototype of a foreign language learning application using mobile augmented reality", Proceedings of the IE 2016 International Conference, vol. 15, Cluj-Napoca, Romania, 2016, pp. 5865 . 


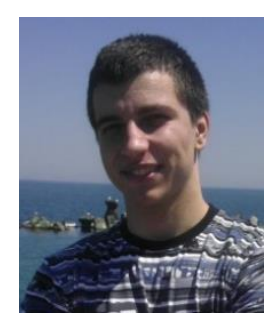

Florentin-Alexandru DIȚĂ has graduated Faculty of Cybernetics, Statistics and Economic Informatics from the Bucharest University of Economic Studies in 2014. He holds a Master diploma in Economic Informatics from 2016. Currently he is PhD student of Economic Informatics at Faculty of Cybernetics, Statistics and Economic Informatics from the Bucharest University of Economic Studies. His work focuses on the mobile programming and analysis of mobile augmented reality systems. 